\title{
Eye Tracking and Pupillometry Are Indicators of Dissociable Latent Decision Processes
}

\author{
James F. Cavanagh \\ Brown University and University of New Mexico
}

\author{
Thomas V. Wiecki, Angad Kochar, \\ and Michael J. Frank \\ Brown University
}

\begin{abstract}
Can you predict what people are going to do just by watching them? This is certainly difficult: it would require a clear mapping between observable indicators and unobservable cognitive states. In this report, we demonstrate how this is possible by monitoring eye gaze and pupil dilation, which predict dissociable biases during decision making. We quantified decision making using the drift diffusion model (DDM), which provides an algorithmic account of how evidence accumulation and response caution contribute to decisions through separate latent parameters of drift rate and decision threshold, respectively. We used a hierarchical Bayesian estimation approach to assess the single trial influence of observable physiological signals on these latent DDM parameters. Increased eye gaze dwell time specifically predicted an increased drift rate toward the fixated option, irrespective of the value of the option. In contrast, greater pupil dilation specifically predicted an increase in decision threshold during difficult decisions. These findings suggest that eye tracking and pupillometry reflect the operations of dissociated latent decision processes.
\end{abstract}

Keywords: decision making, drift rate, decision threshold, pupil, eye tracking

Supplemental materials: http://dx.doi.org/10.1037/a0035813.supp

There is tremendous value in understanding how people reach decisions. Yet it is difficult to objectively characterize the latent processes that contribute to decision making, especially in real time. To understand such processes, simple observable indicators that reflect unobservable decision processes must be identified. In this report, we describe how the observable measures of eye gaze and pupil dilation reflect the operations of dissociated latent systems associated with decision making.

Decision making is often formally modeled by the drift diffusion model (DDM), which provides an algorithmic account of how evidence accumulation and response caution contribute to a binary decision process through the separate latent parameters of drift rate and decision threshold, respectively (Ratcliff, 1978; Ratcliff \& McKoon, 2008). These parameters have orthogonal influences

This article was published Online First February 17, 2014.

James F. Cavanagh, Department of Cognitive, Linguistic, and Psychological Sciences, Brown University, and Department of Psychology, University of New Mexico; Thomas V. Wiecki and Angad Kochar, Department of Cognitive, Linguistic, and Psychological Sciences, Brown University; Michael J. Frank, Department of Cognitive, Linguistic, and Psychological Sciences, Department of Psychiatry, and Brown Institute for Brain Science, Brown University.

The authors thank Laura Kertz for use of the Eyelink system. This project was supported by grants from the National Institutes of Health (5T32MH019118-21 and RO1 MH080066-01) and the National Science Foundation (1125788).

Correspondence concerning this article should be addressed to James F. Cavanagh, Department of Psychology, University of New Mexico, 2001 Redondo Drive, NE, Albuquerque, NM 87131. E-mail: jcavanagh@ unm.edu on the joint measures of accuracy and response time (RT): higher drift captures greater information in the stimulus and results in shorter RT and better accuracy, whereas higher threshold captures increased response caution and results in longer RT and better accuracy. While many investigations use the DDM to account for decision making in noisy sensory environments, this procedure has proved equally valuable to understanding decision making when participants choose between options that have different values, whether values are acquired through learning (Cavanagh et al., 2011) or selected on the basis of preference (Krajbich, Armel, \& Rangel, 2010; Krajbich \& Rangel, 2011).

It has long been known that the decision to select an option out of multiple alternatives can be predicted by gaze dwell time, thought to be a measure of visual attention (Glaholt, Wu, \& Reingold, 2009; Krajbich et al., 2010; Krajbich, Lu, Camerer, \& Rangel, 2012; Krajbich \& Rangel, 2011; Schotter, Gerety, \& Rayner, 2012; Shimojo, Simion, Shimojo, \& Scheier, 2003). This suggests that visual attention has a potentially causal influence on value comparison. Indeed, manipulation of dwell time is associated with increased ventromedial cortical and striatal valuation signals (Lim, O'Doherty, \& Rangel, 2011) and can even influence behavioral selection (Armel, Beaumel, \& Rangel, 2008; Shimojo et al., 2003). This collection of findings has recently been expanded into a model of an attention DDM (aDDM) in which gaze dwell time influences the speed of integrated evidence (drift rate), acting to amplify the inherent value of the fixated relative to nonfixated item (Krajbich et al., 2010, 2012; Krajbich \& Rangel, 2011). However, these findings rely on explicit self-reported ratings of stimulus value, making it difficult to generalize the predictive power this model in other contexts. Moreover, it is unclear whether gaze influences value integration (thus causally affecting 
choice) as in the aDDM, or whether instead gaze might reflect a latent indicator of the participant's endogenously selected choice, in which case it may be predictive independent of value.

Here we extended these findings in two novel ways. First, we utilized controlled and implicit measures of value acquired via history of probabilistic reinforcement to provide a range of positive and negative values. We found that gaze and value both influence drift rate but that the relationships are independent rather than modulatory. Second, we supported the novel hypothesis that pupil dilation reflects an independent latent decision parameter: the need to increase decision threshold when faced with difficult choices.

Most current eye-tracking systems also measure pupil dilation, which is influenced by autonomic nervous system activities. It has long been established that psychological manipulations of orienting, anticipation, fear, cognitive load, arousal, difficulty, anticipation, risk, novelty, surprise, and conflict all cause increases in pupil dilation (Chatham, Frank, \& Munakata, 2009; Goldwater, 1972; Hess \& Polt, 1960; Kahneman \& Beatty, 1966; Laeng, Sirois, \& Gredeback, 2012). Fitting with this large variety of correlated affective and cognitive states, Critchley, Tang, Glaser, Butterworth, and Dolan (2005) discovered that the conflict-related increase in pupil size correlated with activity in perigenual anterior cingulate cortex (ACC). Indeed, the ACC/insula orienting network is implicated in this same broad host of cognitive and affective states, and these neural systems have direct influence over autonomic activities (Craig, 2002; Devinsky, Morrell, \& Vogt, 1995; Shackman et al., 2011). In their role of orienting and signaling the need for control, these neural systems have been structurally (Aron, Behrens, Smith, Frank, \& Poldrack, 2007) and functionally (Cavanagh et al., 2011) implicated as cortical systems that signal the need for increased cognitive control (e.g., raising of the decision threshold) in the face of decision conflict.

We previously demonstrated that frontal midline electroencephalographic (EEG) activities generated by ACC and the surrounding medial wall were specifically implicated in conflict-induced decision threshold adjustment (Cavanagh et al., 2011). While the mechanistic action of threshold increase was initiated by communication between ACC and the subthalamic nucleus, here we propose that pupil dilation may be an observable downstream measure reflecting the ACC "alarm bell" indicating the need for control. The current experiment was therefore designed to test a double dissociation. First, we formally tested for independent and interactive influences of eye gaze dwell time and value on drift rate (Krajbich et al., 2010, 2012; Krajbich \& Rangel, 2011) using hierarchical Bayesian parameter estimation of the DDM. Second, we tested the novel hypothesis that pupil dilation predicts an increase in decision threshold during decision conflict. Findings supported this condition-specific double dissociation in observable measures of latent decision processes.

\section{Method}

\section{Participants}

A total of 24 participants were recruited from the community or the Brown University psychology subject pool to complete the experiment. Participants received either $\$ 20$ or extra course credit for participation. Written informed consent was obtained from all participants. Four participants were excluded due to poor recordings of pupillometry, yielding a total of 20 participants (12 male, average age 20 years).

\section{Probabilistic Selection Task}

Participants performed the probabilistic learning task twice using different nonoverlapping character sets; the assignment between characters and reinforcement probability within each set was randomly assigned (Figure 1A). Data were averaged across both sessions. Each task included a forced-choice training phase in which reinforcement probabilities were acquired, followed by a subsequent testing phase (Frank, Seeberger, \& O'Reilly, 2004). Eye tracking and pupil data were only analyzed from the testing phase, but we describe both parts here for completeness. During the training phase, the participants were presented with a pair of complex visual stimuli (out of three possible pairs), where each choice option (selected with the $d$ and $k$ keyboard keys) was associated with a different probabilistic chance of receiving "correct" or "incorrect" feedback. These stimulus pairs (and their probabilities of reward) were termed A/B (80\%/20\%), C/D (70\%/ $30 \%)$, and $\mathrm{E} / \mathrm{F}(60 \% / 40 \%)$. The participants underwent training trials (consisting of between one and six blocks of 60 stimuli each) until they reached a minimum criterion of choosing the probabilistically best stimulus in each pair: $(\mathrm{A}>\mathrm{B}) \geq 65 \%,(\mathrm{C}>\mathrm{D}) \geq$ $60 \%$, and $(\mathrm{E}>\mathrm{F}) \geq 50 \%$. This same criterion has been used in multiple previous studies with this task. Participants who did not reach this criterion by the end of the sixth block $(n=6$ in the first task, $n=5$ in the second task) were moved to the testing phase regardless.

The testing phase comprised pure decision making: participants chose between all possible pairings of distinct options (presented eight times each; 120 trials per session, two sessions total). Trials began with a fixation cross for $1,000 \mathrm{~ms}$. Stimuli were presented for a maximum of 4,000 ms, and disappeared as soon as a choice was made. Participants were then presented for a blank black screen for $1,000 \mathrm{~ms}$. No feedback was provided in the testing phase. Although no choice between probabilistic stimuli in the test phase is necessarily "correct," hereafter we refer to the selection of the optimal (more reinforcing in the training phase) choice as accuracy.

Conflict trials were defined on the basis of the reinforcement value difference between the available choice options (with smaller, more subtle differences in reinforcement values associated with increasing conflict; see Figure 1A). Thus, we analyzed performance separately for a subset of very high and very low conflict trials, including high-conflict win-win (AC, AE, $\mathrm{CE}$ ), highconflict lose-lose (BD, BF, DF), and low-conflict win-lose (AB, $\mathrm{AD}, \mathrm{AF}, \mathrm{CD}, \mathrm{BC}, \mathrm{BE})$ conditions (Cavanagh, Bismark, Frank, \& Allen, 2012; Cavanagh et al., 2011; Frank, Samanta, Moustafa, \& Sherman, 2007). Prior studies and models suggest that particularly in the win-win conditions, a "hold your horses" mechanism is needed to prevent impulsive responding due to the presence of high-value options, in order to optimize the selection of the most rewarded action (Frank, 2006; Frank et al., 2007). We thus hypothesized that pupil dilation would be reflective of an increase in decision threshold particularly in these trials. For DDM estimations and subsequent empirical comparisons, we parsed these high- 
A

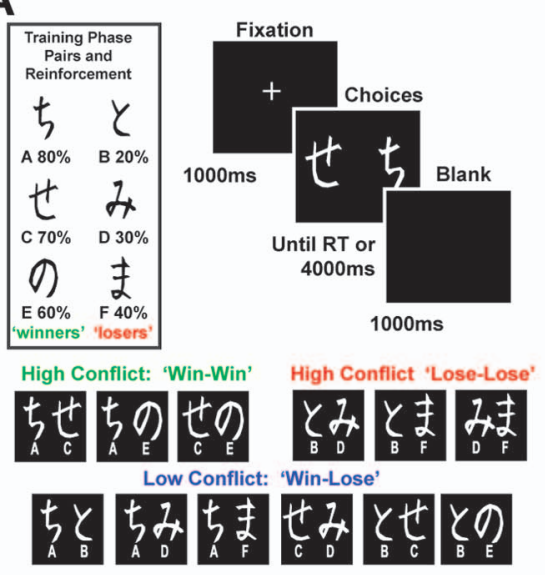

B

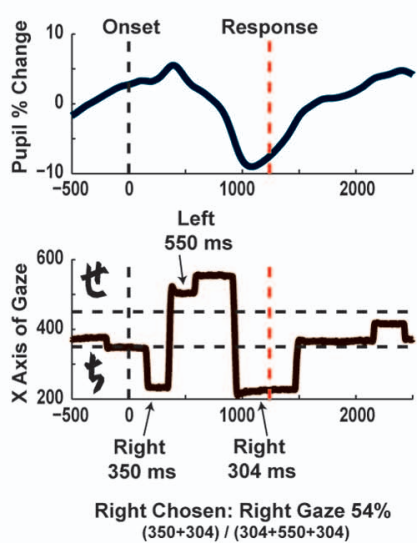

C

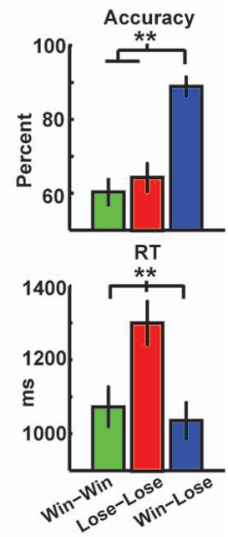

Figure 1. Probabilistic value-based decision-making task, psychophysiological measurement, and performance. (A) During training, each stimulus pair was presented separately. Participants learned to select the better of the two options (the "winner") solely through probabilistic feedback (\% reinforcement is displayed below each stimulus). During the testing phase, each option was paired with all other options, and participants had to choose the better option, without the aid of feedback. Here we investigated high-conflict appetitive (win-win) and aversive (lose-lose) and low conflict (win-lose) conditions within the test phase. (B) Example single trial data for pupil dilation and horizontal eye gaze. Gaze was quantified as a proportional value based on the percentage of dwell time on the optimal stimulus until the choice. (C) As in other studies of this task, participants were more accurate in the easy win-lose condition and were slower in the aversive lose-lose condition. Error bars are standard error of the mean. $\mathrm{ms}=$ milliseconds.

and low-conflict trials into five finer grained categories $(10 \%$, $20 \%, 40 \%, 50 \%$, and $60 \%$ ) corresponding to the difference in the probabilistic values learned during training (i.e., $80 \%-70 \%=$ $10 \%$ for A vs. C, $80 \%-30 \%=50 \%$ for A vs. D, and so on).

To test how gaze dwell time and stimulus value contributed to action selection, we additionally parsed test phase trials into two distinct sets associated with choosing the most rewarding option (defined as the accuracy of choosing A over C, D, E, and F) versus the avoiding the most punishing option (defined as the accuracy of choosing C, D, E, and F over the most punishing symbol, B; Frank et al., 2004). Within each participant, these sets were then split into quantiles based on the proportional amount of gaze time on $\mathrm{A}$ (the best option) or B (the worst option) compared with the other choices (C, D, E, F), facilitating a quantification of the influence of gaze time on stimuli with appetitive (A) versus aversive (B) qualities. While these measures of reward seeking and punishment avoidance vary flexibly within individuals, participants also differ in their general tendency to learn by seeking reward versus avoiding punishment. Individuals more adept at avoidance learning have previously been shown to be more sensitive to negative outcomes and exhibit enhanced error-related brain activity (Cavanagh et al., 2011; Frank, Woroch, \& Curran, 2005). Thus, we also investigated if gaze time influenced selection of aversive stimuli (as predicted by the value-based aDDM) or avoidance of aversive stimuli (e.g., if gaze is predictive independent of value) within a subgroup of participants who were more adept at avoidance learning. These individuals $(n=9)$ were characterized by better accuracy at avoiding the most punishing option over choosing the most rewarding option (as each were defined previously).

\section{Eye Tracking and Pupillometry}

Eye tracking and pupillometry were recorded from an Eyelink 1000 system (SR Research, Kanata, ON, Canada). The Eyelink system was configured using a 35-mm lens, 9-point gaze location calibration, monocular sampling at a rate of $500 \mathrm{~Hz}$, and elliptical fitting for pupil area recordings. Participants were seated approximately $65 \mathrm{~cm}$ away from the eye tracker and performed the study using a stationary head mount. The eye tracker was recalibrated prior to each task. Tasks were presented using the Matlab Psychophysics Toolbox (Mathworks, Natick, MA) and directly interfaced with the eye tracker using the Eyelink application programming interface Psychotoolbox Version 3.0 (Cornelissen, Peters, \& Palmer, 2002).

All data processing was performed using custom Matlab scripts. Pupil measures were taken from the measurement of pupil area, and gaze measures were taken from the $\mathrm{X}$ and $\mathrm{Y}$ coordinates. Blink artifacts detected using the Eyelink blink detection algorithm were removed using linear interpolation from $60 \mathrm{~ms}$ prior to 150 following the event (Siegle, Ichikawa, \& Steinhauer, 2008). If another blink occurred within these interpolation points, all time points between the two blinks were averaged together and the interpolation window was extended. If a blink occurred between fixations within one coordinate window, gaze time was interpolated as the total time including the blink (Krajbich et al., 2010). Most blinks occurred when gazing at the central fixation or when shifting between the stimulus pairs. Continuous data were epoched (from -500 to $4000 \mathrm{~ms}$ ) surrounding the onset of choice option pairs. Each epoch was then visually inspected by modifying the eeglab viewer (Delorme \& Makeig, 2004) to display the time 
course of pupil, gaze, and event detection outputs (saccades, blinks, fixation). Remaining artifacts were user-identified for linear interpolation. Exceedingly noisy conditions were marked for removal. Trials with RTs below $200 \mathrm{~ms}$ or above the RT deadline of 4,000 ms were removed.

Gaze dwell time was measured as the percentage of time that the participant's gaze was on the side of the screen as the optimal choice option (outside of a 100 pixel central vertical buffer between the two sides), divided by the total dwell time in the optimal and suboptimal stimulus sides from presentation until response (Figure 1B). Dwell time on noncentral locations is known to affect pupil dilation by diminishing the size of the pupil in relation to the stationary camera (Brisson et al., 2013; Gagl, Hawelka, \& Hutzler, 2011). We controlled for any potential influence of eye movements by counterbalancing the presentation of the stimulus pairs so each choice option appeared on the right and left sides of the screen an equal number of times. These counterbalancing methods ensured that variations in dwell time, fixations, and saccade timing were uncorrelated with pupil dilation.

Pupil responses were calculated as the percentage of change from the trial-specific prestimulus mean $(-500 \mathrm{~ms}$ to stimulus onset). Stimulus-induced pupil responses begin with a lightinduced constriction and recovery that lasts for about $1 \mathrm{~s}$. In addition, pupil dilations are affected by the act of committing a motor response (Richer \& Beatty, 1985). To account for these issues, all task-specific stimuli were luminance matched and all analyses of high conflict effects used both statistical and subtractive contrasts with the low-conflict (win-lose) condition. Pupil dilations are very slow and thus are lagged in time to eliciting events, often peaking after about 1 s (Gagl et al., 2011; van Steenbergen \& Band, 2013). Therefore, an a priori region of interest for conflict-related pupil dilation was set from 1,000 to 3,000 ms poststimulus All pupil data that were subject to analysis and display were quantified at the resolution of the eye tracker $(500 \mathrm{~Hz})$. For within-subject single trial analyses, pupil size was measured as the mean dilation from the response to $500 \mathrm{~ms}$ following the response, effectively capturing the influences prior to the decision. Given the strong autocorrelation of the pupil response, longer windows provided results highly similar to those reported here. When testing the within-subject single trial relationships between psychophysiological variables (eye gaze, pupil dilation) and performance (RT, accuracy), Spearman's rho and logistic regression were used. Tests of the between-subjects differences in these correlations used $t$ tests and analyses of variance.

\section{Drift Diffusion Model}

The DDM models two-choice decision making as a noisy process accumulating evidence over time (Figure 2). This process approaches one of two boundaries with a certain speed (drift rate, influenced by the amount of evidence conveyed by the stimuli). When one of the two boundaries is crossed, the associated response is executed. The distance between the two boundaries is called the decision threshold; larger thresholds lead to slower but more accurate responding. Estimation of these underlying decision processes was accomplished using DDM analysis of test phase choices. We fit each participant's choices and RT distributions with a DDM model assuming that the proportional difference in reward values for the two options sets the drift rate (Cavanagh et al., 2011; Ratcliff \& Frank, 2012), that is, the rate of evidence accumulation for one option over the other. We used hierarchical

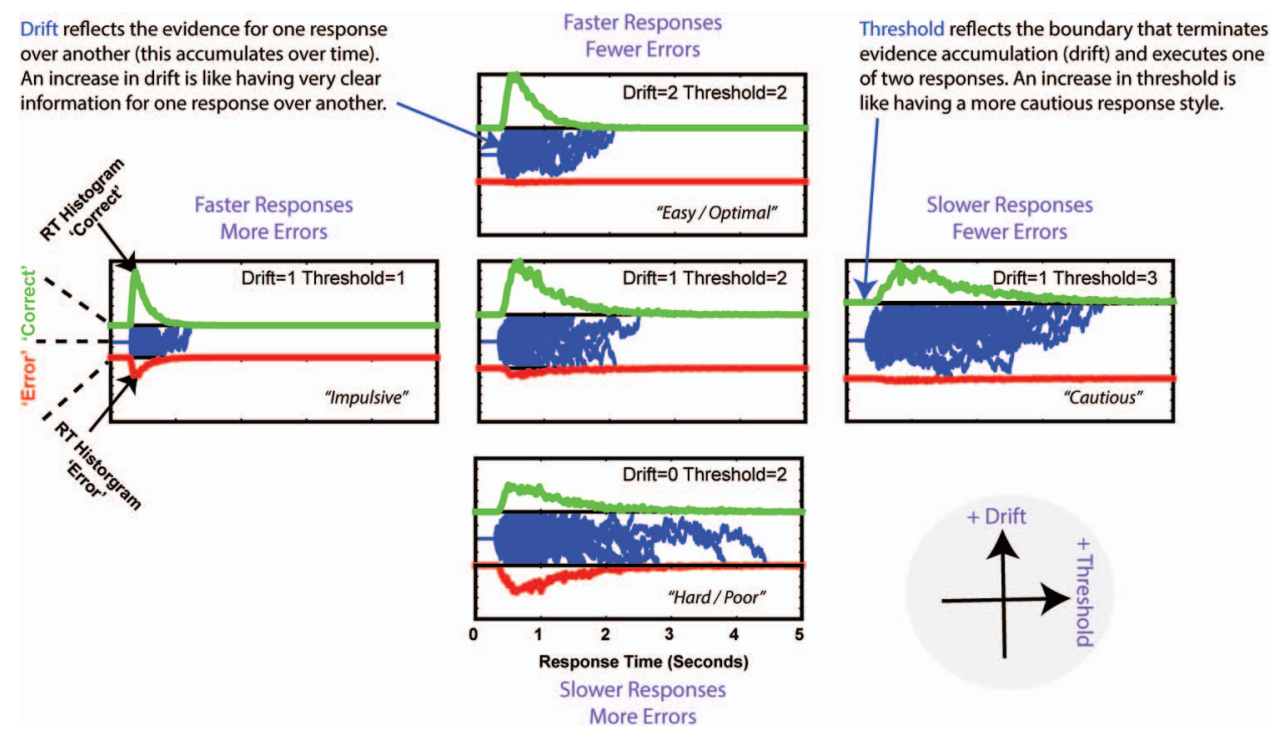

Figure 2. Example of latent drift diffusion model (DDM) parameters. Noisy sensory evidence (blue traces) accumulates toward a bound, whereupon a response is initiated. The duration and direction of these evidence accumulation processes account for the response time (RT) distributions in each of the (binary) conditions (here, correct and erroneous choices are shown). On the vertical axis, an increase in drift rate accounts for increased evidence for one response over another, leading to shorter RTs and better accuracy (bottom toward top). On the horizontal axis an increase in decision threshold raises the boundaries for response execution, leading to longer RTs and better accuracy (left toward right). 
Bayesian estimation of DDM parameters, which optimizes the tradeoff between random and fixed effect models of individual differences, such that fits to individual subjects are constrained by the group distribution but can vary from this distribution to the extent that their data are sufficiently diagnostic (Wiecki, Sofer, \& Frank, 2013). This procedure produces more accurate DDM parameter estimates for individual and groups, particularly given low trial numbers or when assessing coefficients between psychophysiological measures and behavior.

Estimation of the hierarchical DDM (HDDM) was performed using recently developed software (http://ski.clps.brown.edu/ hddm_docs; Wiecki et al., 2013). Bayesian estimation allowed quantification of parameter estimates and uncertainty in the form of the posterior distribution. Markov chain Monte-Carlo (MCMC) sampling methods were used to accurately approximate the posterior distributions of the estimated parameters. Each DDM parameter for each subject and condition was modeled to be distributed according to a normal (or truncated normal, depending on the bounds of parameter intervals) distribution centered around the group mean with group variance. Prior distributions for each parameter were informed by a collection of 23 studies reporting best-fitting DDM parameters recovered on a range of decisionmaking tasks (Matzke \& Wagenmakers, 2009; see the supplement of Wiecki et al., 2013, for visual depictions of these priors). A model using noninformative priors (e.g., uniform distributions that assign equal probability to all parameter values over a large interval) resulted in highly similar results. There were 5,000 samples drawn from the posterior; the first 200 were discarded as burn-in following the conventional approach to MCMC sampling whereby initial samples are likely to be unreliable due to the selection of a random starting point.

We estimated regression coefficients in separate HDDM models to determine the relationship between single trial variations in psychophysiological measures (eye gaze, pupil dilation) and model parameters (drift rate, decision threshold):

$$
\text { parameter }=\beta 0+\beta 1(\text { psychophysiology }) .
$$

In these regressions the coefficient $\beta 1$ weights the slope of parameter (drift rate, threshold) by the value of the psychophysiological measure (proportional gaze dwell time, pupil change from baseline) on that specific trial, with an intercept $\beta 0$. We extended this regression approach to formally compare three competing models of the influence of value and gaze dwell time on drift rate. Each of these models contained multiple regression coefficients in order to test independent and interactive influences of value and gaze dwell time on drift rate. In each of these models, the continuous influence of value $(10 \%, 20 \%, 40 \%, 50 \%$, and $60 \%)$ was used instead of the condition-specific differences (win-win, loselose, and win-lose), although we do plot the condition-specific effect of gaze in one instance for descriptive purposes. For descriptive clarity, these models are formally explained in the Results section. This regression approach was also used to model the influence of pupil dilation on decision threshold in the corrected win-win and lose-lose conditions.

Bayesian hypothesis testing was performed by analyzing the probability mass of the parameter region in question (estimated by the number of samples drawn from the posterior that fall in this region; for example, percentage of posterior samples greater than zero). Statistical analysis was performed on the group mean pos- teriors. The deviance information criterion (DIC) was used for model comparison, where lower DIC values favor models with the highest likelihood and least number of parameters (Gelman, 2004). While alternative methods exist for assessing model fit, DIC is widely used for model comparison of hierarchical models (Spiegelhalter, Best, Carlin, \& van der Linde, 2002), a setting in which Bayes factors are not easily estimated (Wagenmakers, Lodewyckx, Kuriyal, \& Grasman, 2010).

\section{Simulations From Estimated Drift Diffusion Model Parameters}

While the methods described are useful for fitting models to empirical data, it is also important to demonstrate that any estimated combination of model parameters can simulate performance outputs that are qualitatively similar to the empirical inputs that led to the parameter estimation in the first place. First, we contrasted empirical and simulated outcomes to examine the influence of value and gaze duration on action selection. The observed (empirical) probabilities of selecting the optimal stimulus were calculated for the five sets of value differences $(10 \%, 20 \%, 40 \%, 50 \%$, and $60 \%$ ) within each of two different stratifications of the gaze time on the optimal stimulus (high $=z$ score $>0$ vs. low $=z$ score $<$ $0)$. Then synthetic choice probabilities were simulated from the posterior predictive distribution of the best fitting model (independent Model 1, as detailed in the Results) for these same value and gaze stratifications. The simulated values for each of these conditions were each entered into the following formula to predict the probability of selecting the optimal choice based on the analytic solution to the DDM for choice probabilities, where the letters reflect standard notation for DDM parameters: $a=$ decision threshold, $v=$ drift rate, and $z=$ the starting point for the drift process (always at $.5^{*} a$ ):

$$
p(\text { optimal })=\frac{\exp ^{-2^{*} a^{*} z^{*} v}-1}{\exp ^{-2^{*} a^{*} v}-1} .
$$

To simulate the influence of pupil dilation on decision threshold, we generated 50 data sets based on the estimated single trial influence of pupil dilation on decision threshold. The single trial relationships between pupil dilation and simulated/empirical performance (RT, accuracy) were tested using Spearman's correlations and logistic regression, respectively. The mean rho/logistic betas of these 50 simulated sets were then compared. The supplemental materials provide examples and additional descriptive tests of these simulation procedures.

\section{Results}

\section{Probabilistic Selection Task}

As shown in Figure 1C, participants performed similarly as in other experiments with this task (Cavanagh et al., 2011; Frank et al., 2007; Ratcliff \& Frank, 2012). Win-win and lose-lose accuracies were significantly lower than win-lose accuracy, $F(1,19)=$ 27.61, $p<.01$, partial $\eta^{2}=.59, t \mathrm{~s}>5.25, p \mathrm{~s}<.01$, but were not different from each other, $t<1$. Win-win and win-lose RTs were significantly faster than lose-lose RT, $F(1,19)=57.71, p<.01$, 
partial $\eta^{2}=.75 ; t \mathrm{~s}>5.36, p \mathrm{~s}<.01$, but were not different from each other $(t<1)$.

\section{Gaze Dwell Time}

The aDDM predicts a number of relationships between gaze and behavior: (a) the first item fixation should be random with respect to value, (b) the duration of the first fixation should predict selection, (c) the final item fixation before action should be biased toward the chosen item (unless it is has a much lower value than the alternative), and (d) the proportional difference in gaze dwell time should influence choice for equal values of the alternative options (Krajbich et al., 2010, 2012; Krajbich \& Rangel, 2011). Several predictions of this model were validated. The first fixation was unrelated to stimulus value (Figure $3 \mathrm{~A}, p \mathrm{~s}>.22$ ). Within-subject logistic regressions indicated that the amount of time spent gazing on that first item predicted selection in the easiest win-lose condition $(p<.01)$, but not in the harder high-conflict conditions ( $p$ s $>.4$; Figure 3B). The last fixation predicted selection in the win-win and win-lose conditions ( $p$ s $<.01$; Figure 3C), but not in the lose-lose condition $(p=.13)$. Conversely, proportional gaze time strongly predicted selection in all conditions $(p s<.01$; Figure 3D).

To follow up these differences in the propensity of gaze time to predict selection of the fixated option versus selecting the optimal option, we assessed whether gaze effects interacted with the value of choice options. We thus parsed the test phase trials into sets associated with choices involving choosing the most rewarding option versus avoiding the most punishing option (Frank et al., 2004). These sets were then split into quantiles based on the proportional amount of gaze time on A (the best option) or B (the worst option) compared with the other choices $(\mathrm{C}, \mathrm{D}, \mathrm{E}$, and F). Figure $3 \mathrm{E}$ shows that increasing gaze on the optimal A stimulus predicted selection, and thus accuracy, linear trend: $F(1,19)=9.95, p<.01$, partial $\eta^{2}=.34$, whereas increased proportional gaze time on the worst B stimulus also predicted selection, and thus worse accuracy, linear trend: $F(1,19)=$ $75.65, p<.01$, partial $\eta^{2}=.80$. These influences of gaze on selection were found regardless of individual differences in overall learning to choose A versus learning to avoid B. Indeed, even the subgroup of participants more adept at avoidance learning (better at avoiding B than choosing A) still displayed a significant pattern of increasing propensity to select B the more it was observed, $F(1,8)=16.84, p<$ .01 , partial $\eta^{2}=.68$. Thus, increased gaze duration predicted selection even when the option had an aversive value. Note that this pattern contrasts with predictions of the aDDM, which holds that gaze amplifies the value of the fixated proportional to nonfixated item. Hence if this value is aversive, greater gaze time should result in better (not worse) avoidance of that item, as stated in (Armel et al., 2008).

We more formally assess the influence of gaze on value in the context of the DDM quantitative fits later. First, we demonstrate the motivation for this modeling approach with the simple effects supporting the influence of gaze time on drift rate. Figure $4 \mathrm{~A}$ shows group averages of within subject logistic regressions (for accuracy) and nonparametric correlation coefficients (for RTs) as a function of proportional dwell time on the optimal stimulus. Increased dwell time on the optimal stimulus significantly predicted accuracy in all conditions ( $p$ s $<.01$ ), as well as shorter RT, particularly in the easiest win-lose condition $(p<.01)$, fitting with an account of increased drift rate.

\section{Drift Diffusion Modeling With Gaze Dwell Time}

First, to test whether value differences between choice options drive the drift rate, we estimated separate DDM parameters for each condition (Figure 4B). High-conflict win-win and lose-lose conditions (having small value differences) were characterized by much lower drift rates than the low-conflict win-lose condition
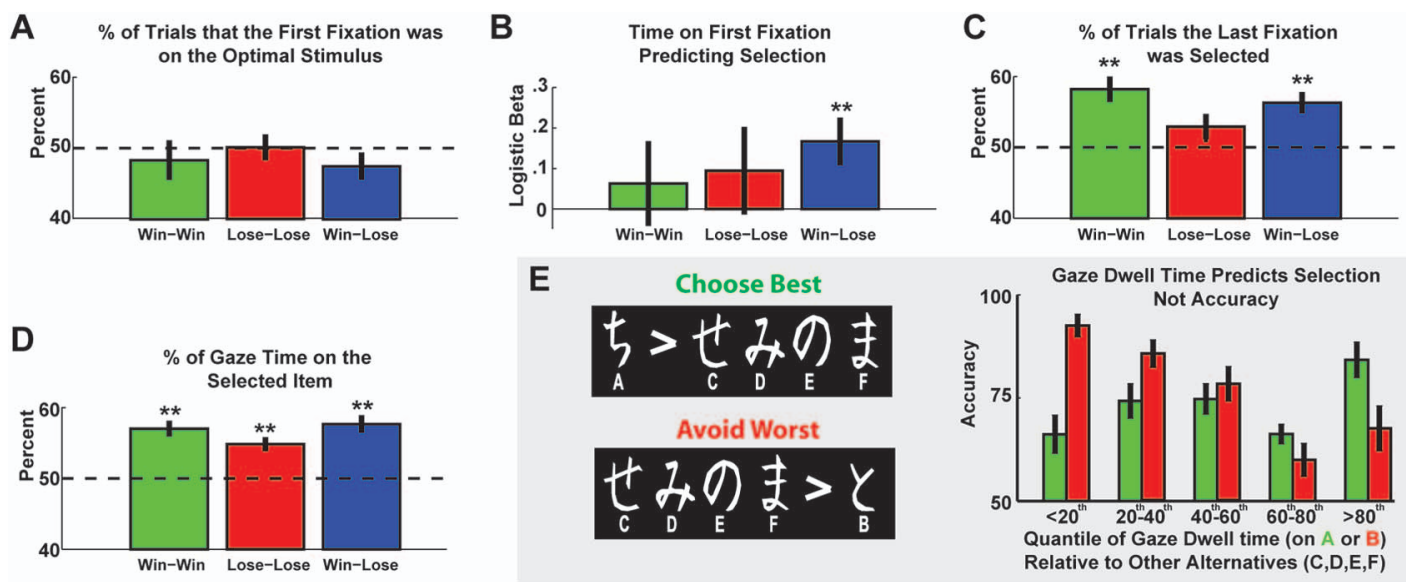

Figure 3. Tests of attention drift diffusion model (aDDM) predictions of the influence of gaze dwell time on selection. The aDDM predicts that (A) the first stimulus fixated upon should be random with respect to option value; (B) the time spent viewing the first option should predict selection; (C) the final item fixated upon should be more likely to be selected; and (D) the relative amount of time gazing on an option should predict selection. E: This panel displays a novel test of whether gaze time interacts with the value of stimuli. Quantiles reflect the amount of relative time viewing the most optimal stimulus (option A) and the worst suboptimal stimulus (option B) compared to the other stimuli (options C-F). Relative viewing time predicted selection in both cases, resulting in improving accuracy for the optimal stimulus but decreasing accuracy for the suboptimal stimulus. Error bars are standard error of the mean. $\mathrm{ms}=$ milliseconds. ${ }^{* *} p<.01$. 
A

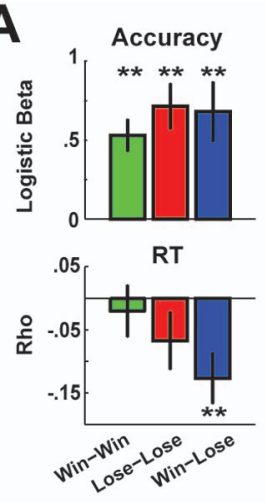

B
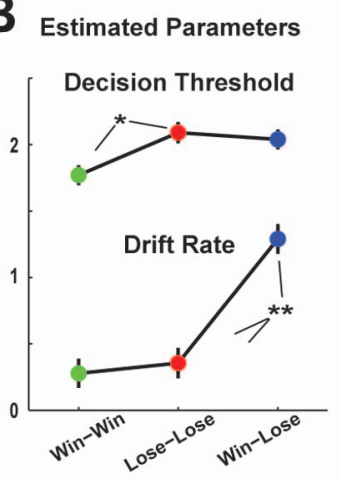

C Effect of Value \& Gaze

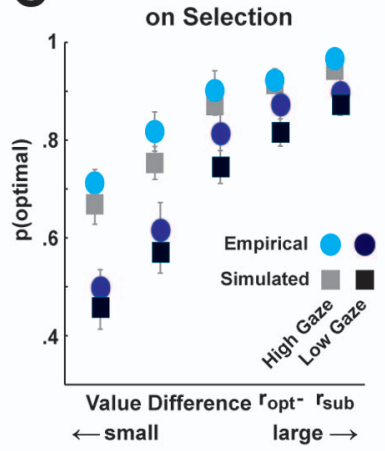

D

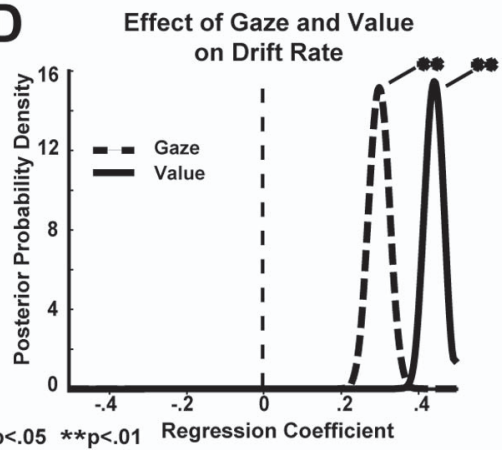

E Condition-Specific Effect of Gaze

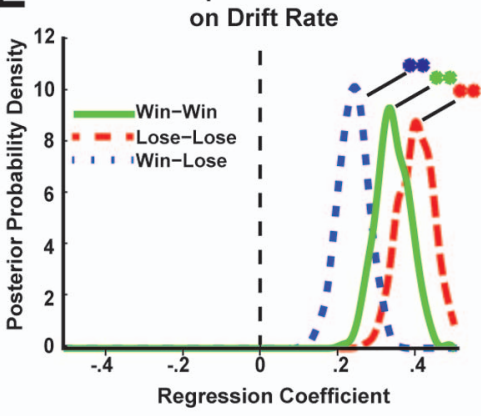

Figure 4. Hierarchical Bayesian parameter estimation of the drift diffusion model (DDM), with gaze time regressors on major decision parameters. (A) Empirical data: relationships between gaze dwell time on the optimal stimulus with accuracy (logistic regression) and reaction time (Spearman's rho). Error bars are standard errors of the mean. (B) Estimated DDM parameters (mean $\pm S D$ ) for each condition. (C) Probability of selecting the optimal choice as a function of value difference between the options, separated by conditions with high versus low gaze durations on the optimal option. Circles are empirical choice (error bars are standard error of the mean across participants), and squares are hierarchical DDM posterior predictive simulations from the independent Model 1 (error bars are SDs of posteriors). (D) Bayesian posterior belief densities from the independent Model 1 of the regression coefficients for value $\left(r_{\mathrm{opt}}-r_{\mathrm{sub}}\right)$ and gaze $\left(\mathrm{gaze}_{\mathrm{opt}}-\right.$ gaze $\left._{\mathrm{sub}}\right)$. (E) Posterior densities of the regression coefficient of gaze on drift rate in a model estimating this effect separately for each condition. Significant effects were determined when $>95 \%$ of the posterior density exceeded 0 .

$(p s<.01)$ but did not differ from each other. The lose-lose condition was also associated with a higher decision threshold than the win-win condition ( $p<.05$ ); see Ratcliff \& Frank, 2012).

These between-condition patterns account for the condition-wide differences in RTs (e.g., slower in lose-lose) and accuracy (e.g., better in win-lose) shown in Figure 1C prior to consideration of the withincondition (single trial) influences of gaze time and pupil dilation on decision parameters. We then estimated regression coefficients in separate HDDM models to determine the relationship between single trial variations in psychophysiological measures and DDM parameters. Model 1 tested for simple independent influences of value and gaze on drift rate $(v)$, where $r_{\text {opt }}$ and $r_{\text {sub }}$ are the reward probabilities of the optimal and suboptimal choices (see Figure 1A), and gaze $\mathrm{opt}_{\mathrm{op}}$

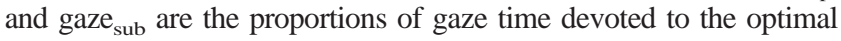
and suboptimal choices on each trial (see Figure 1B):

$$
v=\beta 0+\beta 1 *\left(r_{\mathrm{opt}}-r_{\mathrm{sub}}\right)+\beta 2 *\left(\text { gaze }_{\mathrm{opt}}-\text { gaze }_{\mathrm{sub}}\right)+\varepsilon .
$$

where $\varepsilon$ is a noise term defining intratrial drift. Posterior predictive simulations from this independent Model 1 (Figure 4C squares) closely track the empirical findings (Figure 4C circles), where increasing value differentiation and increased gaze duration predicted selection of the optimal option.

Note that although the model is characterized by independent influences of value and gaze, it still captures the empirical finding of greater effects of gaze when value differences are small. This is a simple geometric property of the DDM where a fixed additive effect has a larger effect when drift rate is low than when it is high (Ratcliff $\&$ McKoon, 2008b). Figure 4D shows the posterior belief densities for these value and gaze terms, revealing strong and statistically significant effects of both variables (see Table 1). For illustrative purposes, we also tested the effect of gaze dwell time on drift rate estimated separately for each value condition (Figure 4E: win-win mean $\beta=$ .35 , lose-lose mean $\beta=.41$, win-lose mean $\beta=.25$, all $p$ s $<.01$ ).

Next, we contrasted alternative formal models of the relationship between gaze time and stimulus value on drift rate. As noted, the aDDM (Krajbich et al., 2010, 2012; Krajbich \& Rangel, 2011) proposes that visual fixation (gaze) on an option is not independent to its value (as in Model 1) but rather influences the degree to which values are integrated toward making a choice. In particular, the aDDM 
Table 1

Deviance Information Criterion Fits and Parameter Values for Each Model of the Influence of Gaze Time and Value on Drift Rate

\begin{tabular}{|c|c|c|c|c|c|c|c|}
\hline \multirow[b]{2}{*}{ Variable } & \multirow[b]{2}{*}{ DIC } & \multirow[b]{2}{*}{$\beta 0$} & \multicolumn{2}{|c|}{$\beta 1$} & \multicolumn{2}{|r|}{$\beta 2$} & \multirow[b]{2}{*}{$\beta 3$ Gaze } \\
\hline & & & Value & Gaze * Value & Gaze & $(1-$ Gaze $) *$ Value & \\
\hline Model 1: Independent & 5795 & $.68(.09)$ & $.44(.02)$ & & $.29(.02)$ & & \\
\hline Model 2: aDDM & 5827 & $.68(.09)$ & & $.72(.03)$ & & $.40(.03)$ & \\
\hline Model 3: aDDM + Gaze & 5809 & $.68(.09)$ & & $.51(.06)$ & & $.58(.05)$ & $.37(.082)$ \\
\hline
\end{tabular}

Note. Mean $(S D)$ regression weights include an intercept $(\beta 0)$ and coefficients $(\beta 1-\beta 3)$ for each separate variable. DIC $=$ deviance information criterion; $\mathrm{aDDM}=$ attention drift diffusion model.

specifies that evidence accumulates as a function of the two decision values according to:

$$
V_{t}=V_{t-1}+d\left(r_{\text {fixated }}-\theta * r_{\text {nonfixated }}\right)+\varepsilon,
$$

where $t$ is the unit of time integrated over, $d$ is a constant that converts values to drift rate, $r\left(_{\text {fixated }}\right)$ and $r\left({ }_{\text {nonfixated }}\right)$ are the relative values of the fixated and nonfixated options, $\theta$ is the degree to which the value of the unfixated item is discounted (from 0 to 1 ), and $\varepsilon$ is noise. Thus, whereas the aDDM in Equation 4 is written in terms of the evolution of the decision variable itself (such that $V_{t}$ depends on $\left.V_{t-1}\right)$, it is straightforward to express this in HDDM terms in which we estimate the drift rate $v$ (the slope with which the decision variable changes):

$$
v=d\left(r_{\text {fixated }}-\theta * r_{\text {nonfixated }}\right)+\varepsilon .
$$

We tested the aDDM in our HDDM framework by indexing the relative time fixated on the optimal vs. suboptimal choice in each trial, and constructing competing models to determine whether gaze time influences drift rate by modulating value (as proposed by the aDDM and Model 2 below), or by simply influencing choice independent of value (Model 1). Note that Equation 5 can be re-expressed to estimate two separate coefficients $\beta 1$ and $\beta 2$ to assess the influence of each choice option value on drift rate:

$$
\begin{aligned}
v & =\beta 0+\beta 1 *\left(\text { gaze }_{\mathrm{opt}} * r_{\mathrm{opt}}-\mathrm{gaze}_{\mathrm{sub}} * r_{\mathrm{sub}}\right) \\
& +\beta 2 *\left(\mathrm{gaze}_{\mathrm{sub}} * r_{\mathrm{opt}}-\mathrm{gaze}_{\mathrm{opt}} * r_{\mathrm{sub}}\right)+\varepsilon,
\end{aligned}
$$

where $d\left(r_{\text {fixated }}-\theta^{*} r_{\text {nonfixated }}\right)$ from Equation 5 is replaced by $\beta 1{ }^{*} r_{\text {fixated }}-\beta 2 * r_{\text {nonfixated }}$, with $\beta 1=d$ and $\beta 2=d^{*} \theta$; hence, $\theta=$ $\beta 2 / \beta 1$. According to the aDDM, $\beta 1>\beta 2$ (i.e., $\theta<1$, indicating that values of fixated options are integrated more than nonfixated ones). Notably, parameter estimates from Model 2 revealed that, indeed, $\beta 1>\beta 2$, consistent with the aDDM prediction that gaze increases value integration. However, as shown in Table 1, Model 2 fit worse (had a higher DIC) than the simple independent Model 1.

To further evaluate whether gaze effects are best described as independent or interactive, we constructed an additional Model 3 that included both interactive terms $(\beta 1, \beta 2)$ and an independent simple additive influence of gaze time $(\beta 3)$ :

$$
\begin{aligned}
v=\beta 0+\beta 1 * & \left(\text { gaze }_{\mathrm{opt}} * r_{\mathrm{opt}}-\mathrm{gaze}_{\mathrm{sub}} * r_{\mathrm{sub}}\right) \\
+\beta 2 *\left(\text { gaze }_{\mathrm{sub}}\right. & \left.r_{\mathrm{opt}}-\text { gaze }_{\mathrm{opt}} * r_{\mathrm{sub}}\right) \\
& +\beta 3 *\left(\mathrm{gaze}_{\mathrm{opt}}-\mathrm{gaze}_{\mathrm{sub}}\right)+\varepsilon
\end{aligned}
$$

Model 3 thus allowed us to assess whether there is a simple effect of gaze $(\beta 3)$ and, if so, whether this alters the conclusions about $\beta 1$ and $\beta 2$ effects of gaze on value. If the effects of gaze and value are truly independent (Model 1), then when fitting Model 3, the $\beta 1$ term should be equal to that of $\beta 2$ because the gaze effects have already been taking into account, implying that we have the standard DDM with drift proportional to value with an additive effect of gaze time.

Indeed, when fitting Model 3 with a pure gaze effect on drift rate, this $\beta 3$ parameter yielded a large regression weight and improved the DIC; the resultant $\beta 1$ and $\beta 2$ were roughly equal (no significant differences in posterior densities, implying that the values of both options were equally integrated). Thus, the initial finding that $\beta 1>\beta 2$ in Model 2 was due to the fact that the $\beta 1$ regressor was correlated with pure gaze differences and that once the pure gaze effect was taken into account, the resulting value component amounts effectively to the pure DDM without gaze modulation of value (i.e., $\beta 1 \sim \beta 2, \theta \sim 1$; see Method). This conclusion, based on parameter estimation, was confirmed by the observation that Model 1, including simple independent contributions of pure value $\left(r_{\mathrm{opt}}-r_{\mathrm{sub}}\right)$ and pure gaze $\left(\mathrm{gaze}_{\mathrm{opt}}-\mathrm{gaze}_{\mathrm{sub}}\right)$, provided the best fit to the data from a model selection standpoint.

These findings were highly similar when tested on all test phase trials (not just win-win, lose-lose, and win-lose choices as defined in Figure 1), and they were clearly stronger than any potential influence of gaze time on decision threshold (all DICs $>6,320$ for Models 1-3 predicting threshold instead of drift rate). Therefore, participants may select poorly valued options (such as "B") when looking at them more, since the influence of gaze dwell time on selection is independent - not interactive - with stimulus value.

\section{Pupil Response}

Figures $5 \mathrm{~A}$ and $5 \mathrm{~B}$ show stimulus and response-locked pupil dilation as a percentage of change from prestimulus baseline. There was a significant increase in the a priori stimulus-locked region of interest as revealed by a repeated-measures analysis of variance-from 1,000 - to 3,000-ms poststimulus: $F(1,19)=7.74$, $p<.05$, partial $\eta^{2}=.07$. Follow-up paired $t$ tests revealed that win-win trials had significantly larger pupil dilations than winlose trials, $t(19)=3.53, p<.01$, with lose-lose trials falling between these extremes-win-win $>$ lose-lose: $t(19)=1.66, p=$ .11 ; lose-lose $>$ win-lose: $t(19)=2.18, p=.07$. For illustrative purposes, significant differences between each pair of conditions are shown over the time course of the pupil response. Figures $5 \mathrm{C}$ and 5D shows relationships between pupil dilation and RT (time point by time point correlation coefficients) and pupil dilation and accuracy (pupil difference in optimal minus suboptimal choices). These single trial correlations revealed a complex pre- and postre- 


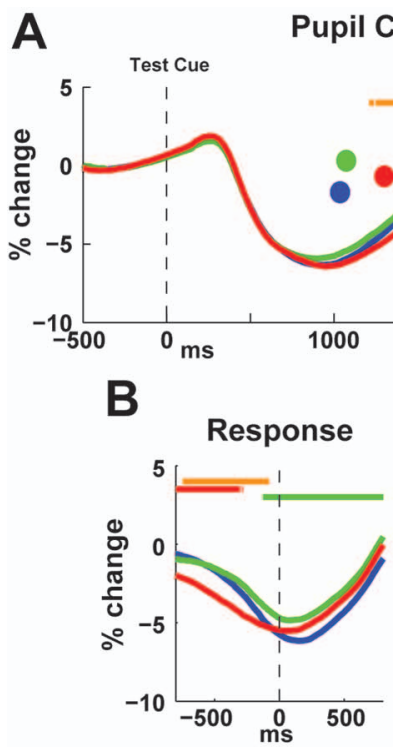

D

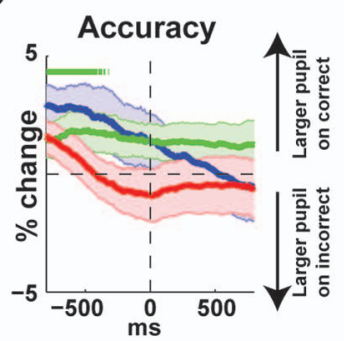

Corr with RT Control for WL

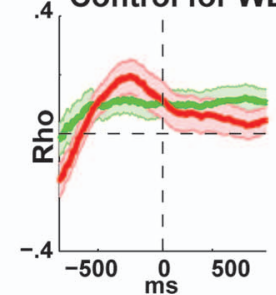

F Effect of Pupil Dilation on Decision Threshold

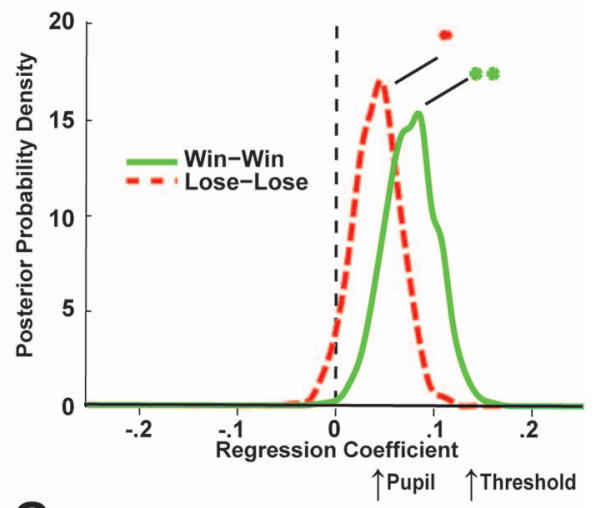

G
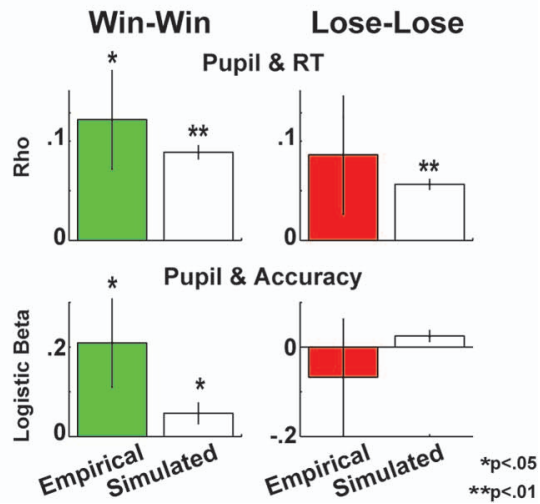

Figure 5. Phasic pupil change and relation to decision threshold. (A) Pupil change locked to the test phase presentation of choice options. The initial peak and subsequent trough are due to the pupil light reflex (from $\sim 100$ to $1,000 \mathrm{~ms}$ ). Dots indicate mean response times (RTs) for each condition. Horizontal bars indicate time points that were significantly different between specified conditions. (B) Response-locked pupil dilation. (C) Timepoint-by-timepoint Spearman's correlations between response-locked pupil dilation and RT. Error bars are standard error of the mean (SEM). (D) Pupil dilation on correct minus incorrect choices. Error bars are SEM. (E) Correlations are as in (C) but are corrected for canonical influences in high-conflict conditions by subtraction of low-conflict correlations. (F) Posterior of the regression coefficients for pupil dilation on decision threshold in the win-win (WW) and lose-lose (LL) high-conflict conditions (corrected by subtraction of low-conflict pupil dilation). Significant effects were determined when $>95 \%$ of the posterior density exceeded 0 . (G) Empirical (color) and simulated posterior predictive (white) relationships between pupil dilation and performance (RT and accuracy).

sponse relationship between pupil change and RT in all conditions. This pattern was due to the influence of the light and response reflexes. Thus, we needed to account for these canonical responses. Sample-by-sample subtractions of the win-lose correlation from the win-win and lose-lose correlations revealed a constant influence of pupil dilation on RT (Figure 5E). Notably, postresponse pupil dilation was associated with longer RTs and better accuracy, particularly in the win-win condition, a pattern consistent with an increase of the decision threshold during high conflict choices.

\section{Drift Diffusion Modeling With Pupil Dilation}

In contrast to the effect of gaze on drift rate, HDDM revealed significant posterior belief distributions for the effect of pupil dilation on decision threshold for high-conflict conditions. This model of decision threshold fit the empirical data (DIC: 4387) better than an alternative model investigating the influence of pupil dilation on drift rate (DIC: 4396). Conflict-specific effects were highlighted using subtraction of the average win--lose condition from single trial win-win and lose-lose trials. Figure $5 \mathrm{~F}$ shows the significant effects for the conflict-specific influence of pupil dilation on decision threshold (win-win mean $\beta=$ $.075, p<.01$; lose-lose mean $\beta=.04, p<.05$ ). These same findings were also replicated in a more temporally sensitive analysis with statistical contrasts against the win-lose condition (instead of subtraction), demonstrating the robustness of this effect (see supplemental online materials). Posterior predictive simulations yielded estimated RTs and accuracies from this model that estimated single trial changes in decision threshold as a function of pupil dilation (Figure 5G). Larger pupil dilation 
correlated with slower RTs and better accuracy in the win-win condition (all empirical and simulated $p \mathrm{~s}<.05$ ), but effects in the lose-lose condition were varied (only simulated RT significantly correlated with pupil dilation, $p<.01$ ). These simulations indicate that the trial-by-trial effect of pupil dilation on decision threshold can be accurately estimated, particularly for high-conflict appetitive conditions.

\section{Discussion}

The findings reported here support the hypothesis that eye gaze and pupil dilation reflect dissociated biases in latent decision parameters of evidence accumulation (drift rate) and response caution (decision threshold). These findings suggest that it is possible to predict variance in distinct decision processes simply by observing the eyes of a subject.

\section{Gaze Time and Evidence Accumulation}

The evidence reported here suggests that the influences of value and gaze time have independent influences on drift rate when choices are based on experienced, implicit valuation. While many of the predictions of the aDDM (Krajbich et al., 2010, 2012; Krajbich \& Rangel, 2011) were confirmed here, evidence from psychophysical contrasts, model comparisons, and model parameters suggest that the aDDM overspecifies the multiplicative influence of gaze time on value as related to drift rate. We found that increased gaze dwell time can predict selection independent of value, even when gaze is focused on the least positive (or most aversive) option.

The aDDM directly suggests that longer gaze times on a negative value should lead to increased rejection (Armel et al., 2008), which was not observed. This was most strongly demonstrated by the increasing relationship of gaze time on the most aversive B stimulus and selection (leading to poorer accuracy), even in a subgroup of participants who learned the task with increased ability to avoid B over seeking A. Thus, the influence of gaze time on selecting B cannot be attributed to poor learning of its value. Instead, these findings support the notion that better avoidance of aversive stimuli is also reflected by looking away from the aversive items. However, this interpretation is predicated on the assumption that the B stimulus is aversive and not just of low value. Nevertheless, even if the B option had a positive but simply lower value than other options, the aDDM would still predict that the effect of gaze would be diminished in the lose-lose condition compared with the win-win condition, given the smaller multiplicative influence of lower values (see the online supplemental material for a detailed example). Figure $4 \mathrm{E}$ demonstrates that the HDDM reveals the opposite of this prediction.

The findings reported here differ from a prior study (Armel et al., 2008), which showed that participants were more likely to avoid an aversive food item if it had been presented for a longer period of time than an alternative option-but critically these options appeared sequentially. Our findings from this forcedchoice experiment suggest that when participants fixate on aversive or low-value options, they still may be more likely to actively avoid those options by choosing to look at and, ultimately, select the other option. Yet, if instead they dwell on the low-value option for a longer period of time, they are more likely to choose it.
It is useful to consider how the valenced forced-choice conditions used here may lead to different findings than those observed previously. It may be expected that the win-win and lose-lose conditions should have identical findings, given that the relative value differences are identical for each constituent trial pair. Yet as revealed by simple performance differences (Figure 1C), appetitive and aversive conflict differ in a number of meaningful ways. First, it has been long known that RTs are slower in this lose-lose condition (Cavanagh et al., 2012; Frank et al., 2007; Ratcliff \& Frank, 2012). This slowing is thought to be a result of the compounded influence of two aversive responses, which produce additional slowing via the corticostriatal indirect pathway, in part by amplifying the subthalamic response to conflict (Frank et al., 2004; Ratcliff \& Frank, 2012). This slowing is captured by an increased decision threshold (Figure 4B). Moreover, the cognitive strategies that contribute to successful test-phase execution differ between selection (win-win) and avoidance (lose-lose). One example of this difference may be revealed by the tendency of the last fixation to predict selection in all conditions except lose-lose (Figure 3C) due to active avoidance rather than appetitive seeking. It remains possible that while proportional gaze duration influences evidence accumulation and option selection in all conditions (Figure 3D), eye movements characterized by volitional saccades may contribute to different aspects of cognitive strategies during avoidance versus reward seeking.

Formal model comparison and parameter estimation approaches provided converging evidence that value differences directly influence drift rate (as in the simple DDM) and that the effect of gaze is an independent influence on drift rate. Previous reports of the aDDM only included choices between appetitive options and, hence, could not easily differentiate a model in which gaze directly influences drift rather than modulates the values, which are confounded (Krajbich et al., 2010, 2012; Krajbich \& Rangel, 2011). A more recent example of the aDDM during complicated purchasing decisions revealed a much smaller multiplicative effect of value and gaze $(\theta=0.7$, closer to standard DDM), clearly demonstrating that task dynamics have a strong influence on the strength and existence of this phenomenon (Krajbich et al., 2012).

\section{Pupillometry and Response Caution}

The dynamics of pupil dilation have previously been quantified using numerous formal modeling approaches, including learning rate (Nassar et al., 2012), prediction error (Preuschoff, 't Hart, \& Einhäuser, 2011), neural gain (Eldar, Cohen, \& Niv, 2013), and salience in decision making (Fiedler \& Glöckner, 2012), but none have investigated a potential role in decision threshold adjustment. This event-specific dynamic analysis of decision threshold is a relatively novel advancement in models of two-alternative forced choice, supported by previous neurobiological studies and DDM modeling (Cavanagh et al., 2011; Ratcliff \& Frank, 2012). How does the participant decide to increase the decision threshold upon seeing a difficult choice? According to computational models, premotor areas of cortex (including ACC) rapidly detect the strength of stimulus-response links based on past history, and when the two response options have similar frequencies, this conflict evokes a fast signal via the corticosubthalamic "hyperdirect" pathway to raise the decision threshold (Ratcliff \& Frank, 2012). In parallel, the striatum accumulates noisy value signals for 
the two options. Thus, the system can raise the threshold for difficult choices based on response heuristics before detecting the relative value differences.

In this study, appetitive (win-win) decision conflict was most strongly associated with increased pupil dilation, which itself was associated with longer RT, better accuracy, and increased decision threshold. The apparent sensitivity of these effects to appetitive conflict likely relate to the greater requirement for cognitive control in this situation to overcome the otherwise impulsive tendency to choose either good option (Cavanagh et al., 2011; Frank et al., 2007) - that is, action invigoration associated with positive value would otherwise act to reduce the effective decision threshold. Nevertheless, aversive decision conflict (lose-lose) did have a smaller but statistically significant relationship between pupil dilation and increased decision threshold (Figure 5F), despite the larger intercept (Figure 4B). These findings are consistent with previous observations that high-conflict appetitive or aversive choices are associated with increased decision threshold and with increased mediofrontal signatures of conflict (Cavanagh et al., 2011), thus lending support to the notion that pupil dilation may reflect downstream processing of ACC activities.

Future studies may also aim to test this effect using very long decision periods or neutral luminance changes in order to obviate the influence of the pupil light reflex. Given that the pupil light reflex is parasympathetically mediated, whereas task-relevant dilations are sympathetically mediated (Beatty \& Lucero-Wagoner, 2000), trial-specific variance is likely to be unrelated to reflexive activities and is thus recoverable (Wierda, van Rijn, Taatgen, \& Martens, 2012). In this study, the win-win and win-lose conditions were characterized by highly similar RTs, allowing post hoc subtraction or regression, but these procedures may not have been as effective for the lose-lose condition, which had reliably longer RTs. Regardless, considerable evidence suggests that pupil dilation to appetitive decision conflict was clearly associated with longer RTs, better accuracy, and increased decision threshold.

\section{Future Directions and Implications}

These findings suggest that the addition of eye gaze and pupil dilation measures would be beneficial to consider in future investigations of neural systems involved in evidence accumulation (Gold \& Shadlen, 2007; Hare, Schultz, Camerer, O’Doherty, \& Rangel, 2011; O'Connell, Dockree, \& Kelly, 2012) and decision threshold adjustment (Cavanagh et al., 2011; Domenech \& Dreher, 2010; Forstmann et al., 2010). Such methodological integration will be necessary to test the independent and potentially causal nature of gaze time on action selection. For example, the interpretation of the relationships between observable variables and latent effects described here may not be reflective of the neuronal processes contributing to either of these facets, as neuronal inputoutput conversions are likely to be nonlinear (thus eliminating the difference between additive vs. multiplicative effects; Wagenmakers, Krypotos, Criss, \& Iverson, 2012).

The diffusion model is but one particular instance of a class of sequential sampling models of decision making. Although alternative models differ in some details, all involve a single decision boundary and accumulated evidence over time. Given the large similarities and equivalence under certain conditions (Bogacz, Brown, Moehlis, Holmes, \& Cohen, 2006; Donkin, Brown, Heath- cote, \& Wagenmakers, 2011), we predict that similar conclusions would likely be drawn if relating the psychophysiological measures to other related models, such as the linear ballistic accumulator model (Brown \& Heathcote, 2008) and the leaky competing accumulator model (Usher \& McClelland, 2001).

Eye tracking is already heavily utilized in fields as diverse as human-computer interaction (tobii.com; theeyetribe.com), website evaluation (gazehawk.com), marketing (invivo-bva.com; imotionsglobal.com), driver safety (sixsafetysystems.com), and highperformance training (polhemus.com; eyecomcorp.com). Yet simultaneous metrics of pupil dilation have not been commercially utilized to the same extent as eye gaze, perhaps due to low specificity (as noted in the introduction). However, commercial enterprises have begun integrating eye tracking with measurements of facial musculature (realeyesit.com) and observational pulse monitoring (vitalsignscamera.com), suggesting that such an additional independent metric would be highly desirable if the information content of the signal could be reliably determined. Indeed, simultaneous eye tracking and pupillometry have already been utilized for understanding complex behavioral economic interactions (Wang, Spezio, \& Camerer, 2010).

\section{Conclusion}

We suggest that continued investigation of eye tracking and pupillometry using formal computational models will enhance the predictive sensitivity and specificity of these observable states. The outcome of such investigations will not only be fruitful for the study of psychological science but also may contribute to technological innovations for varied commercial and public health applications.

\section{References}

Armel, K. C., Beaumel, A., \& Rangel, A. (2008). Biasing simple choices by manipulating relative visual attention. Judgment and Decision Making, 3, 396-403.

Aron, A. R., Behrens, T. E., Smith, S., Frank, M. J., \& Poldrack, R. A. (2007). Triangulating a cognitive control network using diffusionweighted magnetic resonance imaging (MRI) and functional MRI. Journal of Neuroscience, 27, 3743-3752. doi:10.1523/JNEUROSCI.0519-07 .2007

Beatty, J., \& Lucero-Wagoner, B. (2000). The pupillary system. In J. T. Cacioppo, L. G. Tassinary, \& G. G. Berntson (Eds.), Handbook of psychophysiology (2nd ed., pp. 142-162). Cambridge, England: Cambridge University Press.

Bogacz, R., Brown, E., Moehlis, J., Holmes, P., \& Cohen, J. D. (2006). The physics of optimal decision making: A formal analysis of models of performance in two-alternative forced-choice tasks. Psychological Review, 113, 700-765. doi:10.1037/0033-295X.113.4.700

Brisson, J., Mainville, M., Mailloux, D., Beaulieu, C., Serres, J., \& Sirois, S. (2013). Pupil diameter measurement errors as a function of gaze direction in corneal reflection eyetrackers. Behavior Research Methods, 45, 1322-1331. doi:10.3758/s13428-013-0327-0

Brown, S. D., \& Heathcote, A. (2008). The simplest complete model of choice response time: Linear ballistic accumulation. Cognitive Psychology, 57, 153-178. doi:10.1016/j.cogpsych.2007.12.002

Cavanagh, J. F., Bismark, A. J., Frank, M. J., \& Allen, J. J. B. (2012). Larger error signals in major depression are associated with better avoidance learning. Frontiers in Psychology, 2, 331. doi:10.3389/fpsyg .2011 .00331 
Cavanagh, J. F., Wiecki, T. V., Cohen, M. X., Figueroa, C. M., Samanta, J., Sherman, S. J., \& Frank, M. J. (2011). Subthalamic nucleus stimulation reverses mediofrontal influence over decision threshold. Nature Neuroscience, 14, 1462-1467. doi:10.1038/nn.2925

Chatham, C. H., Frank, M. J., \& Munakata, Y. (2009). Pupillometric and behavioral markers of a developmental shift in the temporal dynamics of cognitive control. Proceedings of the National Academy of Sciences, USA, 106, 5529-5533. doi:10.1073/pnas.0810002106

Cornelissen, F. W., Peters, E. M., \& Palmer, J. (2002). The Eyelink Toolbox: Eye tracking with MATLAB and the Psychophysics Toolbox. Behavior Research Methods, Instruments, \& Computers, 34, 613-617.

Craig, A. D. (2002). How do you feel? Interoception: The sense of the physiological condition of the body. Nature Reviews Neuroscience, 3, 655-666. doi:10.1038/nrn894

Critchley, H. D., Tang, J., Glaser, D., Butterworth, B., \& Dolan, R. J. (2005). Anterior cingulate activity during error and autonomic response. NeuroImage, 27, 885-895. doi:10.1016/j.neuroimage.2005.05.047

Delorme, A., \& Makeig, S. (2004). EEGLAB: An open source toolbox for analysis of single-trial EEG dynamics including independent component analysis. Journal of Neuroscience Methods, 134, 9-21.

Devinsky, O., Morrell, M. J., \& Vogt, B. A. (1995). Contributions of anterior cingulate cortex to behaviour. Brain, 118, 279-306.

Domenech, P., \& Dreher, J.-C. (2010). Decision threshold modulation in the human brain. The Journal of Neuroscience, 30, 14305-14317. doi: 10.1523/JNEUROSCI.2371-10.2010

Donkin, C., Brown, S., Heathcote, A., \& Wagenmakers, E.-J. (2011). Diffusion versus linear ballistic accumulation: Different models but the same conclusions about psychological processes? Psychonomic Bulletin \& Review, 18, 61-69. doi:10.3758/s13423-010-0022-4

Eldar, E., Cohen, J. D., \& Niv, Y. (2013). The effects of neural gain on attention and learning. Nature Neuroscience, 16, 1146-1153. doi: $10.1038 / \mathrm{nn} .3428$

Fiedler, S., \& Glöckner, A. (2012). The dynamics of decision making in risky choice: An eye-tracking analysis. Frontiers in Psychology, 3, 335. doi:10.3389/fpsyg.2012.00335

Forstmann, B. U., Anwander, A., Schafer, A., Neumann, J., Brown, S., Wagenmakers, E. J., . . Turner, R. (2010). Cortico-striatal connections predict control over speed and accuracy in perceptual decision making. Proceedings of the National Academy of Sciences, USA, 107, 1591615920. doi:10.1073/pnas.1004932107

Frank, M. J. (2006). Hold your horses: A dynamic computational role for the subthalamic nucleus in decision making. Neural Networks, 19, $1120-1136$

Frank, M. J., Samanta, J., Moustafa, A. A., \& Sherman, S. J. (2007, November 23). Hold your horses: Impulsivity, deep brain stimulation, and medication in Parkinsonism. Science, 318, 1309-1312. doi:10.1126/ science. 1146157

Frank, M. J., Seeberger, L. C., \& O’Reilly, R. C. (2004, December 10). By carrot or by stick: Cognitive reinforcement learning in Parkinsonism. Science, 306, 1940-1943. doi:10.1126/science.1102941

Frank, M. J., Woroch, B. S., \& Curran, T. (2005). Error-related negativity predicts reinforcement learning and conflict biases. Neuron, 47, 495501. doi:10.1016/j.neuron.2005.06.020

Gagl, B., Hawelka, S., \& Hutzler, F. (2011). Systematic influence of gaze position on pupil size measurement: Analysis and correction. Behavior Research Methods, 43, 1171-1181. doi:10.3758/s13428-011-0109-5

Gelman, A. (2004). Bayesian data analysis (Texts in statistical science series, 2nd ed., p. xxv, 668 p.). Boca Raton, FL: Chapman \& Hall/CRC.

Glaholt, M. G., Wu, M., \& Reingold, E. M. (2009). Predicting preference from fixations. PsychNology Journal, 7, 141-158.

Gold, J. I., \& Shadlen, M. N. (2007). The neural basis of decision making. Annual Review of Neuroscience, 30, 535-574. doi:10.1146/annurev .neuro.29.051605.113038
Goldwater, B. C. (1972). Psychological significance of pupillary movements. Psychological Bulletin, 77, 340-355. doi:10.1037/h0032456

Hare, T. A, Schultz, W., Camerer, C. F., O’Doherty, J. P., \& Rangel, A. (2011). Transformation of stimulus value signals into motor commands during simple choice. Proceedings of the National Academy of Sciences, USA, 108, 18120-18125. doi:10.1073/pnas. 1109322108

Hess, E. H., \& Polt, J. M. (1960, August 5). Pupil size as related to interest value of visual stimuli. Science, 132, 349-350. doi:10.1126/science.132 .3423 .349

Kahneman, D., \& Beatty, J. (1966, December 23). Pupil dilation and load on memory. Science, 154, 1583-1585. doi:10.1126/science.154.3756 .1583

Krajbich, I., Armel, C., \& Rangel, A. (2010). Visual fixations and the computation and comparison of value in simple choice. Nature Neuroscience, 13, 1292-1298. doi:10.1038/nn.2635

Krajbich, I., Lu, D., Camerer, C., \& Rangel, A. (2012). The attentional drift-diffusion model extends to simple purchasing decisions. Frontiers in Psychology, 3, 193. doi:10.3389/fpsyg.2012.00193

Krajbich, I., \& Rangel, A. (2011). Multialternative drift-diffusion model predicts the relationship between visual fixations and choice in valuebased decisions. Proceedings of the National Academy of Sciences, USA, 108, 13852-13857. doi:10.1073/pnas.1101328108

Laeng, B., Sirois, S., \& Gredeback, G. (2012). Pupillometry: A window to the preconscious? Perspectives on Psychological Science, 7, 18-27. doi:10.1177/1745691611427305

Lim, S.-L., O'Doherty, J. P., \& Rangel, A. (2011). The decision value computations in the vmPFC and striatum use a relative value code that is guided by visual attention. The Journal of Neuroscience, 31, 1321413223. doi:10.1523/JNEUROSCI.1246-11.2011

Matzke, D., \& Wagenmakers, E.-J. (2009). Psychological interpretation of the ex-Gaussian and shifted Wald parameters: A diffusion model analysis. Psychonomic Bulletin \& Review, 16, 798-817. doi:10.3758/PBR .16 .5 .798

Nassar, M. R., Rumsey, K. M., Wilson, R. C., Parikh, K., Heasly, B., \& Gold, J. I. (2012). Rational regulation of learning dynamics by pupillinked arousal systems. Nature Neuroscience, 15, 1040-1046. doi 10.1038/nn.3130

O'Connell, R. G., Dockree, P. M., \& Kelly, S. P. (2012). A supramodal accumulation-to-bound signal that determines perceptual decisions in humans. Nature Neuroscience, 15, 1729-1735. doi:10.1038/nn.3248

Preuschoff, K., 't Hart, B. M., \& Einhäuser, W. (2011). Pupil dilation signals surprise: Evidence for noradrenaline's role in decision making. Frontiers in Neuroscience, 5, 115. doi:10.3389/fnins.2011.00115

Ratcliff, R. (1978). A theory of memory retrieval. Psychological Review, 85, 59-108. doi:10.1037/0033-295X.85.2.59

Ratcliff, R., \& Frank, M. J. (2012). Reinforcement-based decision making in corticostriatal circuits: Mutual constraints by neurocomputational and diffusion models. Neural Computation, 24, 1186-1229. doi:10.1162/ NECO_a_00270

Ratcliff, R., \& McKoon, G. (2008a). The diffusion decision model: Theory and data for two-choice decision tasks. Neural Computation, 20, 873922. doi:10.1162/neco.2008.12-06-420

Richer, F., \& Beatty, J. (1985). Pupillary dilations in movement preparation and execution. Psychophysiology, 22, 204-207. doi:10.1111/j.14698986.1985.tb01587.x

Schotter, E. R., Gerety, C., \& Rayner, K. (2012). Heuristics and criterion setting during selective encoding in visual decision-making: Evidence from eye movements. Visual Cognition, 20, 1110-1129. doi:10.1080/ 13506285.2012 .735719

Shackman, A. J., Salomons, T. V., Slagter, H. A., Fox, A. S., Winter, J. J., \& Davidson, R. J. (2011). The integration of negative affect, pain and cognitive control in the cingulate cortex. Nature Reviews Neuroscience, 12, 154-167. doi:10.1038/nrn2994 
Shimojo, S., Simion, C., Shimojo, E., \& Scheier, C. (2003). Gaze bias both reflects and influences preference. Nature Neuroscience, 6, 1317-1322. doi:10.1038/nn1150

Siegle, G. J., Ichikawa, N., \& Steinhauer, S. (2008). Blink before and after you think: Blinks occur prior to and following cognitive load indexed by pupillary responses. Psychophysiology, 45, 679-687. doi:10.1111/j 1469-8986.2008.00681.x

Spiegelhalter, D. J., Best, N. G., Carlin, B. P., \& van der Linde, A. (2002). Bayesian measures of model complexity and fit. Journal of the Royal Statistical Society: Series B (Statistical Methodology), 64, 583-639. doi:10.1111/1467-9868.00353

Usher, M., \& McClelland, J. L. (2001). The time course of perceptual choice: The leaky, competing accumulator model. Psychological Review, 108, 550-592. doi:10.1037/0033-295X.108.3.550

van Steenbergen, H., \& Band, G. P. H. (2013). Pupil dilation in the Simon task as a marker of conflict processing. Frontiers in Human Neuroscience, 7, 215. doi:10.3389/fnhum.2013.00215

Wagenmakers, E.-J., Krypotos, A.-M., Criss, A. H., \& Iverson, G. (2012). On the interpretation of removable interactions: A survey of the field 33 years after Loftus. Memory \& Cognition, 40, 145-160. doi:10.3758/ s13421-011-0158-0
Wagenmakers, E.-J., Lodewyckx, T., Kuriyal, H., \& Grasman, R. (2010) Bayesian hypothesis testing for psychologists: A tutorial on the SavageDickey method. Cognitive Psychology, 60, 158-189. doi:10.1016/j .cogpsych.2009.12.001

Wang, J. T., Spezio, M., \& Camerer, C. F. (2010). Pinocchio's pupil: Using eyetracking and pupil dilation to understand truth-telling and deception in games. American Economic Review, 3, 984-1007. doi 10.1257/aer.100.3.984

Wiecki, T. V., Sofer, I., \& Frank, M. J. (2013). HDDM: Hierarchical Bayesian estimation of the drift-diffusion model in Python. Frontiers in Neuroinformatics, 7, 14-24. doi:10.3389/fninf.2013.00014

Wierda, S. M., van Rijn, H., Taatgen, N. A., \& Martens, S. (2012). Pupil dilation deconvolution reveals the dynamics of attention at high temporal resolution. Proceedings of the National Academy of Sciences, USA, 109, 8456-8460. doi:10.1073/pnas.1201858109

Received August 26, 2013

Revision received January 1, 2014

Accepted January 5, 2014 